\title{
Gating attosecond pulse train generation using multicolor laser fields
}

\author{
H.-C. Bandulet, ${ }^{1}$ D. Comtois, ${ }^{1}$ E. Bisson, ${ }^{1}$ A. Fleischer, ${ }^{2}$ H. Pépin, ${ }^{1}$ J.-C. Kieffer, ${ }^{1}$ P. B. Corkum, ${ }^{2}$ and D. M. Villeneuve ${ }^{2}$ \\ ${ }^{1}$ Institut National de la Recherche Scientifique, 1650 Boulevard Lionel-Boulet, Varennes, Québec J3X 1S2, Canada \\ ${ }^{2}$ National Research Council of Canada, 100 Sussex Drive, Ottawa, Ontario K1A OR6, Canada
}

(Received 15 April 2009; published 5 January 2010)

\begin{abstract}
The process of high-order harmonic generation leads to the production of a train of attosecond-duration extreme ultraviolet (XUV) pulses, with one pulse emitted per optical half-cycle. For attosecond pump-probe experiments, a single, isolated attosecond pulse is preferable, requiring an almost continuous spectrum. We show experimentally and numerically that the addition of a second laser field, and later a third, at a noncommensurate frequency relative to the driving field can modify the subcycle shape of the electric field, leading to the appearance of additional spectral components between the usual odd harmonics and in some cases a quasicontinuum. We perform a parametric study of the frequency ratio between the two first laser fields, the result of which is in good agreement with theoretical selection rules. We also show numerically that using three laser frequencies from an optical parametric amplifier can achieve a single attosecond pulse from a 24-fs laser pulse.
\end{abstract}

DOI: 10.1103/PhysRevA.81.013803

PACS number(s): 42.65.Ky, 42.65.Re, 42.65.Yj

\section{INTRODUCTION}

Attosecond pulses (AP) [1] are a powerful new tool to probe and manipulate matter on the time scale of electron motion $[2,3]$. They are most commonly produced via highorder harmonic generation (HHG) of an intense laser field in a gas medium, in which one short XUV pulse is emitted during every half-cycle of the driving field. A typical multicycle driver pulse will produce a train of AP. Although AP trains can be applied in their own right [4-7], great effort has been expended in recent years to isolate single AP to permit a wider range of pump-probe measurements.

The key principle to decrease the number of AP in the train is to reduce the periodicity of the HHG process until the emission is confined to a very short time interval. In the frequency domain, this amounts to the generation of a spectral supercontinuum [8,9], which can be achieved by reducing the number of cycles of the driver [10] and/or by introducing a gate or switch of subcycle duration to longer pulses. The shortest single AP produced so far, lasting less than 100 as, have been generated from 3.3-fs pulses [11]. These approaches also rely on spectral filtering of AP to select emission from only the highest intensity part of the pulse, as well as ionization gating to terminate the process.

However, few-cycle pulses are technically challenging to produce $[12,13]$. Therefore, other approaches are being pursued to relax the need to produce heroically short driving pulses. For example, polarization gating (PG) in combination with few-cycle pulses, which has recently yielded 130-as pulses [14], exploits the high sensitivity of the recollision step to the field's ellipticity [15] by rapidly sweeping the polarization state of the field from circular to linear within one optical cycle [14,16-18]. Another technique has been demonstrated in which the addition of a phase-locked second harmonic field [19-22] inhibits the ionization on one polarity of the optical field and causes the HHG spectrum to show a mixture of even and odd harmonics. This results in an AP train with a separation of a full optical period. Combined with PG in a technique called double optical gating, an XUV supercontinuum capable of supporting 130-as pulses was generated using 9-fs driving pulses [23]. Other approaches of combining different frequencies have been studied theoretically [24-26]. A recent experiment demonstrated increased spectral density when two incommensurate laser frequencies were combined [27], creating the conditions necessary for temporal confinement of attosecond pulse generation.

In this article, we experimentally demonstrate the feasibility of a new gating technique that relies on the mixing of two or three multicycle laser pulses at incommensurate frequencies to further modify the subcycle shape of the electric field in order to produce single AP. The mixing of incommensurate driving frequencies for the purpose of synthesizing single AP has only been studied theoretically [24,28] but has never been realized in practice until now. Previous experiments used this approach to achieve tunability of HHG [27,29,30]. Our results clearly show that the addition of a second (and then a third) optical field whose frequency is not commensurate with the primary field can create additional frequency components in the HHG spectrum. Depending on the frequency ratio between the fields, this can result in a quasicontinuous spectrum in the plateau region. We also show numerically that this subcycle field control will lead to the possibility of generating a single attosecond pulse from a combination of three 24-fs laser pulses, similar to a proposal by [31] to combine three regions of a broadband spectrum to produce a single-cycle pulse.

\section{EXPERIMENTAL DETAILS}

The experiment was performed with the 100-Hz Ti:Sa laser system (Thales) at the Advanced Laser Light Source (ALLS) in Quebec. Approximately $6 \mathrm{~mJ}$ at $800 \mathrm{~nm}$ was directed into an optical parametric amplifier (OPA) (TOPAS-HE from Light Conversion) and $0.7 \mathrm{~mJ}$ bypassed the OPA. The 800 -nm driver frequency entering the OPA was frequency-converted into a signal and an idler beam, the wavelengths of which were adjustable between 1200 and $1550 \mathrm{~nm}$ and 1650 and $2050 \mathrm{~nm}$, respectively. The OPA maximum pulse energy after steering was $1.0 \mathrm{~mJ}$ at $1300 \mathrm{~nm}$ and $0.6 \mathrm{~mJ}$ at $2020 \mathrm{~nm}$ with a pulse duration of $80 \mathrm{fs}$. The energy and polarization state of the $800-\mathrm{nm}$ and signal beams were controlled by half-wave plates and polarizers (achromatic in the 1000-1600 $\mathrm{nm}$ range for the signal). Neutral density filters were used to reduce the energy 

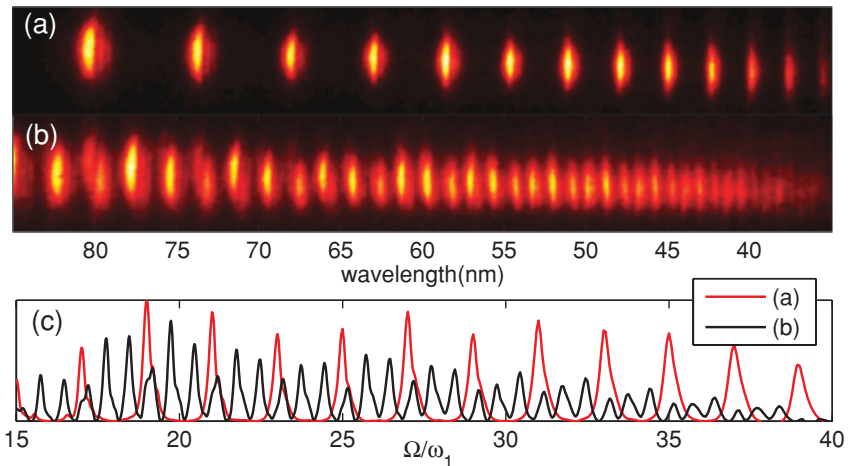

FIG. 1. (Color online) Typical experimental high-order harmonic spectra generated in argon with an OPA wavelength of $1400 \mathrm{~nm}$ $\left(I \approx 3 \times 10^{14} \mathrm{~W} / \mathrm{cm}^{2}\right)$ in (a), showing only odd harmonic orders, and with the addition of a weak 800 -nm field $\left(I \approx 10^{13} \mathrm{~W} / \mathrm{cm}^{2}\right)$ in (b). (c) Spectra shown in (a)(red or gray line) and (b)(black line) after integration in the vertical direction. $\Omega$ and $\omega_{1}$ are the XUV and driving frequencies, respectively.

of the idler. The relative polarization state of all three beams was made parallel for the results shown. The relative timing of the beams was adjusted using motorized delay stages with a 1 -fs resolution. The signal and idler beams were recombined with up to $100 \mu \mathrm{J}$ of bypassed 800-nm light using dichroic mirrors. Collinearity of the beams was achieved by superposing their far-field images using an IR-Vidicon camera. The beams were focused by a $f=250 \mathrm{~mm}$ spherical mirror into a thin pulsed gas jet backed with a pressure of 3 atm of either argon or krypton. The $800 \mathrm{~nm}$ was kept below the intensity at which it could generate harmonics by itself. The laser intensities were estimated using the method described in [32]. Using a fast ion detector (modified beam dynamics model Fig. 1, see [32] for details) placed below the gas jet, the position of the laser focus could be precisely controlled and placed $2 \mathrm{~mm}$ before the center of the jet to select short electron trajectories. The harmonic emission, collimated in the direction of the driving beams, entered a flat-field XUV spectrometer composed of a slit, concave grating, and a microchannel plate (MCP) coupled to a phosphor readout screen that was then imaged onto a cooled 14-bit CCD camera.

\section{RESULTS AND DISCUSSION}

In Fig. 1 we show typical spectra generated in argon with a laser field at $1400 \mathrm{~nm}$ combined with a weaker 800-nm pulse. In Fig. 1(a) the two beams are in space but not in time; hence only the odd harmonics of the 1400-nm field are present. When the beams are temporally overlapped as well, two additional frequency components of intensities comparable to those of the odd-order peaks become evident in Fig. 1(b), thus multiplying the conversion efficiency in a given wavelength range, which in this particular case amounts to a $40 \%$ increase in the 40-80 nm range.

Fleischer and Moiseyev [28] used a Floquet approach to analyze the symmetry of the HHG process with two laser fields and have determined a simple selection rule to determine the various pathways to a particular emitted frequency. They
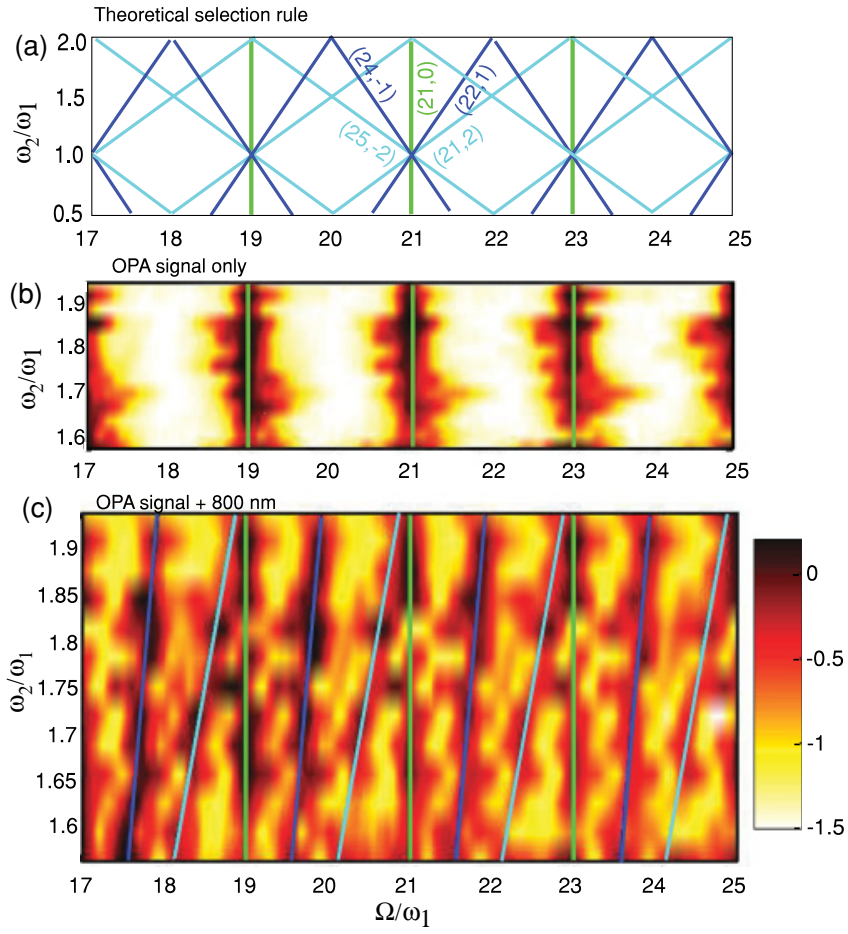

FIG. 2. (Color online) (a) Schematic of the positions of the frequency components predicted by the model of Fleischer and Moiseyev [28]. (b, c) Experimentally recorded XUV spectra from the HHG in argon using two driving frequencies: $\omega_{1}$ is the IR driving field $\left(I \approx 10^{14} \mathrm{~W} / \mathrm{cm}^{2}\right.$ at all wavelengths) and $\omega_{2}$ is the weaker $800-\mathrm{nm}$ field $\left(I \approx 10^{13} \mathrm{~W} / \mathrm{cm}^{2}\right)$. Each horizontal line is the spectrum for a particular OPA signal wavelength $\left(\omega_{1}\right)$. Panel $(b)$ has only the OPA wavelength, while panel (c) has both fields simultaneously present. The color (shading) scale is logarithmic in intensity. The lines from the model have been superimposed for $n_{2}=0,+1$, and +2 .

conclude that an emitted XUV frequency $\Omega$ can be produced by various numbers of photons $\left(n_{1}, n_{2}\right)$ from the two laser fields $\left(\omega_{1}, \omega_{2}\right)$ :

$$
\Omega=n_{1} \omega_{1}+n_{2} \omega_{2}, \quad n_{1}+n_{2}=2 k-1, \quad n_{1}, n_{2}, k \in Z .
$$

For comparison with the experimental results, we choose $\omega_{1}$ to be our strong near infrared frequency, and $\omega_{2}$ to be the weaker $800-n m$ frequency. In Fig. 2(a) the lines show the predicted frequencies based on this model for three orders of the 800-nm photon, $n_{2}=0$ (green vertical lines), 1 (dark blue slightly tilted lines), and 2 (light blue tilted lines). The horizontal axis is the emitted XUV frequency divided by the intense OPA laser frequency $\omega_{1}$, whereas the vertical axis is the $800-\mathrm{nm}$ frequency $\omega_{2}$ divided by $\omega_{1} . n_{2}=0$ corresponds to the usual single-frequency case which gives the odd harmonics of $\omega_{1}$. The higher-order processes become increasing less likely because $\omega_{2}$ is relatively weak compared with $\omega_{1}$. For the case $\omega_{2}=2 \omega_{1}$, the $n_{2}= \pm 1$ selection rule leads to even harmonics, as has been observed when mixing the second harmonic of the driving field [20-22]. At all OPA wavelengths, we observed additional frequency components in the HHG spectrum when the weak 800 -nm field was simultaneously present. We show in Figs. 2(b) and 2(c) the spectra for OPA wavelengths in the range $1200-1550 \mathrm{~nm}$ with and without the 

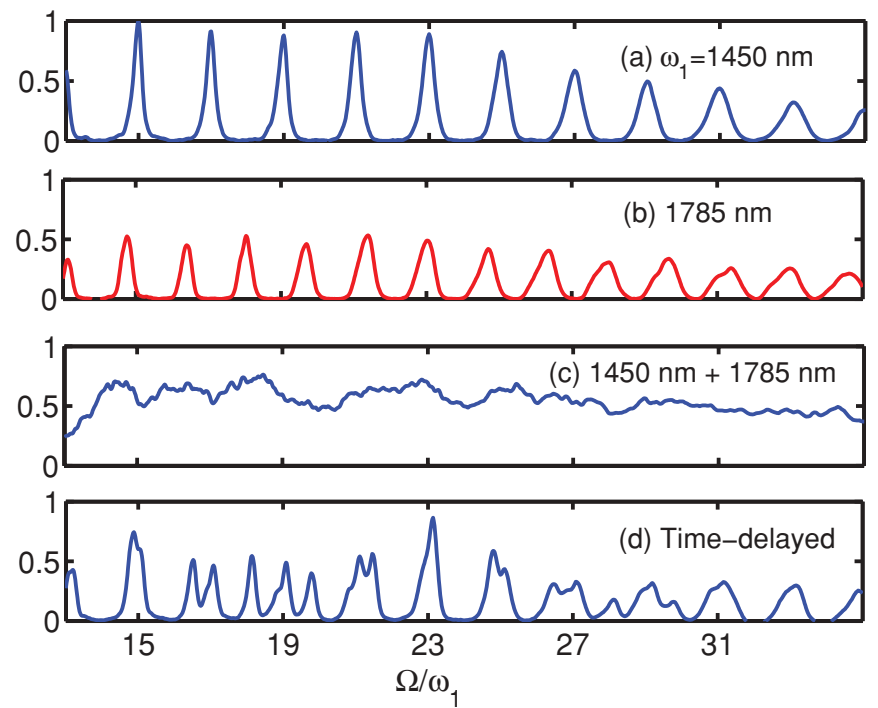

FIG. 3. (Color online) HHG spectra produced in krypton by (a) the signal at $1450 \mathrm{~nm}\left(I \approx 3 \times 10^{14} \mathrm{~W} / \mathrm{cm}^{2}\right)$, (b) the idler at $1785 \mathrm{~nm}\left(I \approx 1.5 \times 10^{14} \mathrm{~W} / \mathrm{cm}^{2}\right)$, (c) the two beams simultaneously present, and then (d) delayed in time by $200 \mathrm{fs}$. The vertical scales are shown relative to (a). Here, $\omega_{2} / \omega_{1}=0.81$, that is, close to the case where $\omega_{2} \rightarrow \omega_{1}$ for which the model predicts a dense spectrum consisting of many hyper-Raman lines.

perturbation. Only the positive $n_{2}$ orders seem to be present. For example between harmonics 21 and 23 we see $\left(n_{1}, n_{2}\right)=$ $(20,1),(19,2)$, but not $(24,-1),(25,-2)$. The negative terms correspond to higher-order processes, for example, $(19,2)$ versus $(25,-2)$, which have lower probabilities, and may explain why they are not visible in the experiment.

Although it is not shown here, we have also experimentally investigated the intensity dependence of the second field. The intensity of the $n_{2}=1$ frequency component grows linearly with the intensity of the 800-nm field up to an energy of $20 \mu \mathrm{J}$, then saturates. The linear growth is consistent with the weak field contributing one photon.

The Floquet picture [28] predicts that the density of peaks will increase as the two laser frequencies become closer to one another, that is, $\omega_{2} \rightarrow \omega_{1}$. We have experimentally investigated the special case where $\omega_{2} / \omega_{1}=0.81$, by combining the OPA signal at $1450 \mathrm{~nm}$ and the idler at $1785 \mathrm{~nm}$ at intensities where they each produce their own set of odd-order harmonics, as shown in Fig. 3. When the two beams are spatially combined but don't overlap in time, the two spectra are simply superimposed on the detector. When the beams are made coincident in time, the generated harmonic spectrum becomes very rich with extra components [Fig. 3(c)].

What about the relative phase between the different driving frequency components? The Floquet model predicts the location of the additional frequency components based on the addition and subtraction of photons, which therefore suggests that their positions should be independent of the relative phase. This is confirmed in our experiment, in which the different laser frequencies are not interferometrically stabilized-the peaks appear at the positions predicted by the Floquet model even though the relative phase varies between laser pulses.
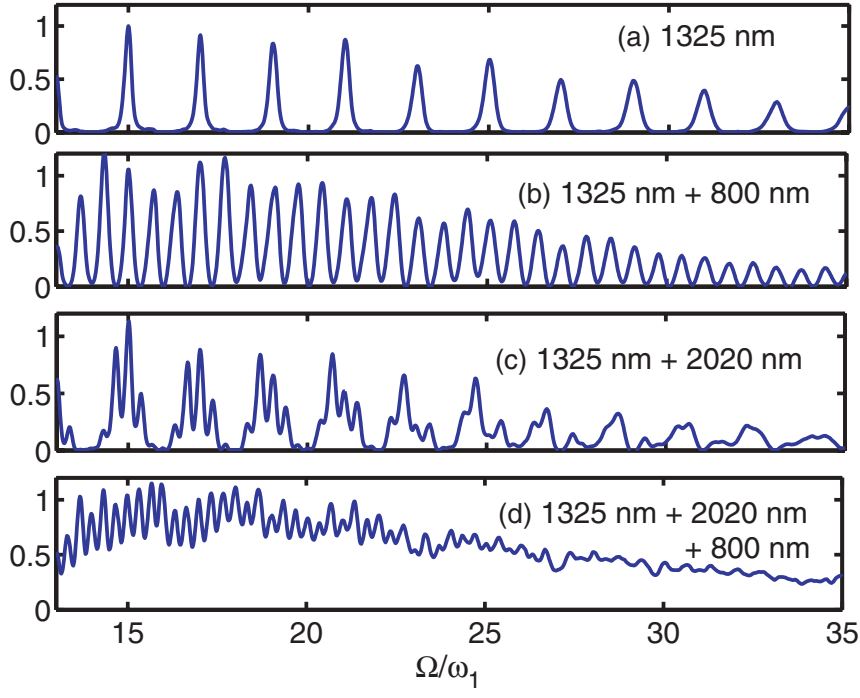

FIG. 4. (Color online) HHG spectra generated in krypton by the OPA signal beam at $1325 \mathrm{~nm}\left(I \approx 3 \times 10^{14} \mathrm{~W} / \mathrm{cm}^{2}\right)$ in (a). The OPA signal is combined with (b) $20 \mu \mathrm{J}$ at $800 \mathrm{~nm}\left(I \approx 10^{13} \mathrm{~W} / \mathrm{cm}^{2}\right)$, (c) $100 \mu \mathrm{J}$ at $2020 \mathrm{~nm}\left(I \approx 10^{13} \mathrm{~W} / \mathrm{cm}^{2}\right)$, and (d) both 800 and $2020 \mathrm{~nm}$. The HHG amplitude is shown relative to (a).

There is interest in using longer driver wavelengths to produce attosecond pulses, since the cutoff frequency scales as $I \lambda^{2}$ [33]. The HHG spectra when combining three wavelengths (OPA signal plus idler plus pump) is shown in Fig. 4, where we have added weak fields at 800 and $2020 \mathrm{~nm}$ to a strong harmonic-generating signal at $1325 \mathrm{~nm}$. The spectrum becomes quasicontinuous. Because our OPA is seeded by superfluorescence, the three frequencies are not phase-locked in the present experiment. Therefore, combining them cannot yield isolated AP. However, OPAs that use white light generation to produce the seed for the signal wavelength can have all three frequencies phase-locked [34]. It is then possible to produce a single, isolated AP from a multicycle pulse.

To demonstrate this idea, in Fig. 5 we show a calculation in which 800-, 1300-, and 2080-nm fields, each with a full width at half maximum duration (FWHM) of $24 \mathrm{fs,} \mathrm{are} \mathrm{added}$ together. The subcycle shape of the wave form results in only one electron recollision event that leads to the emission of a single attosecond pulse. The dotted line shows the prediction of a time-dependent Schrodinger calculation using the electric field shape shown in the figure. The model predicts that only a single attosecond pulse will be produced, even though we started with a 24-fs pulse. This technique does not require the use of capillary pulse compressors, chirped mirrors, or polarization gating techniques. It only requires that the three outputs from an OPA be combined with the appropriate phase. This is similar to calculations [25] in which a slightly detuned second harmonic was added to the fundamental field, leading to an isolated attosecond pulse. Our present method gives better contrast because of the reduced periodicity of the electric field and does not rely on depletion to reduce post-pulses. This approach requires a stable phase relationship between the different frequency components, and the relative phases must be adjusted to deliver a single attosecond pulse as shown in Fig. 5. The adjustment of the relative phase between frequency 


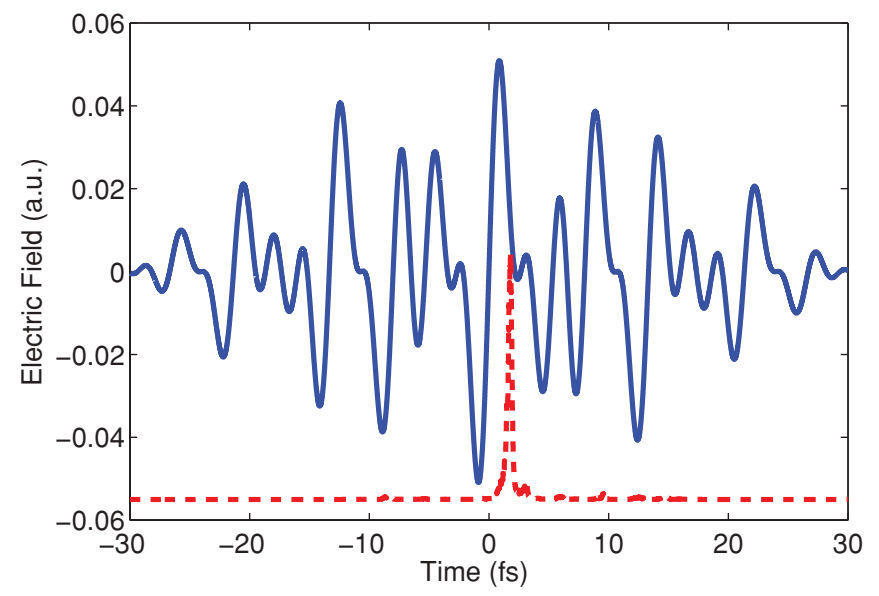

FIG. 5. (Color online) The electric field resulting from the sum of three fields corresponding to 800,1300 , and $2080 \mathrm{~nm}$. The last two wavelengths represent the signal and idler of an OPA pumped by $800 \mathrm{~nm}$. Each field has a Gaussian envelope with an intensity FWHM of $24 \mathrm{fs}$. The field is now sufficiently aperiodic that a single attosecond pulse will be generated at the peak of the envelope. The dashed line shows (shifted vertically) the predicted XUV intensity as calculated with a time-dependent Schrodinger equation model with a smoothed hydrogen atom potential. Each electric field had an amplitude of 0.02 a.u. The relative phase was such that all three are sines at the peak of the envelope, the optimum condition for generating an isolated pulse. The XUV was filtered for photons greater than $50 \mathrm{eV}$. Propagation of the fields was not included. components has already been done for the double optical gating technique [23] and is accomplished by rotating a glass plate.

\section{CONCLUSION}

In summary, we have experimentally investigated the feasibility of a new gating technique to produce single attosecond pulses by combining two or three multicycle pulses at incommensurate frequencies from the output of an optical parametric amplifier. We demonstrate that the frequency ratio between the fields determines the number and location of additional sidebands that appear in the spectrum. We also show that when two beams have similar frequencies or when three infrared beams are combined, the spectrum becomes quasicontinuous and able to support light bursts of very short duration. Time-dependent Schrödinger calculations show that this method could yield single attosecond pulses from the combination of three relatively long pulses of $24 \mathrm{fs}$ in duration.

\section{ACKNOWLEDGMENTS}

The authors thank the ALLS team for support and L. Andrzejewski for developing the data acquisition and motion control software. We gratefully acknowledge support from NSERC and AFOSR.
[1] F. Krausz and M. Ivanov, Rev. Mod. Phys. 81, 163 (2009).

[2] M. Drescher, M. Hentschel, R. Kienberger, M. Uiberacker, V. Yakovlev, A. Scrinzi, T. Westerwalbesloh, U. Kleineberg, U. Heinzmann, and F. Krausz, Nature (London) 419, 803 (2002).

[3] A. L. Cavalieri et al., Nature (London) 449, 1029 (2007).

[4] P. Johnsson et al., Phys. Rev. Lett. 95, 013001 (2005).

[5] T. Remetter et al., Nat. Phys. 2, 323 (2006).

[6] K. Varjú et al., J. Phys. B 39, 3983 (2006).

[7] J. Mauritsson, P. Johnsson, E. Mansten, M. Swoboda, T. Ruchon, A. L'Huillier, and K. J. Schafer, Phys. Rev. Lett. 100, 073003 (2008).

[8] B. Shan, S. Ghimire, and Z. Chang, J. Mod. Opt. 52, 277 (2005).

[9] Y. Peng and H. Zeng, Phys. Rev. A 78, 033821 (2008).

[10] M. Hentschel, R. Kienberger, C. Spielmann, G. A. Reider, N. Milosevic, T. Brabec, P. Corkum, U. Heinzmann, M. Drescher, and F. Krausz, Nature (London) 414, 509 (2001).

[11] E. Goulielmakis et al., Science 320, 1614 (2008).

[12] M. Nisoli, S. D. Silvestri, and O. Svelto, Appl. Phys. Lett. 68, 2793 (1996).

[13] M. Nisoli, S. D. Silvestri, O. Svelto, R. Szipöcs, K. Ferencz, C. Spielmann, S. Sartania, and F. Krausz, Opt. Lett. 22, 522 (1997).

[14] G. Sansone et al., Science 314, 443 (2006).

[15] K. S. Budil, P. Salières, A. L'Huillier, T. Ditmire, and M. D. Perry, Phys. Rev. A 48, R3437 (1993).

[16] O. Tcherbakoff, E. Mével, D. Descamps, J. Plumridge, and E. Constant, Phys. Rev. A 68, 043804 (2003).
[17] A. Zaïr, O. Tcherbakoff, E. Mével, E. Constant, R. LópezMartens, J. Mauritsson, P. Johnsson, and A. L'Huillier, Appl. Phys. B 78, 869 (2004).

[18] I. Sola et al., Nat. Phys. 2, 319 (2006).

[19] K. Kondo, Y. Kobayashi, A. Sagisaka, Y. Nabekawa, and S. Watanabe, J. Opt. Soc. Am. B 13, 424 (1996).

[20] N. Dudovich, O. Smirnova, J. Levesque, Y. Mairesse, M. Y. Ivanov, D. M. Villeneuve, and P. B. Corkum, Nat. Phys. 2, 781 (2006).

[21] I. J. Kim, C. M. Kim, H. T. Kim, G. H. Lee, Y. S. Lee, J. Y. Park, D. J. Cho, and C. H. Nam, Phys. Rev. Lett. 94, 243901 (2005).

[22] J. Mauritsson, P. Johnsson, E. Gustafsson, A. L'Huillier, K. J. Schafer, and M. B. Gaarde, Phys. Rev. Lett. 97, 013001 (2006).

[23] H. Mashiko, S. Gilbertson, C. Li, S. D. Khan, M. M. Shakya, E. Moon, and Z. Chang, Phys. Rev. Lett. 100, 103906 (2008).

[24] T. Pfeifer, L. Gallmann, M. J. Abel, P. M. Nagel, D. M. Neumark, and S. R. Leone, Phys. Rev. Lett. 97, 163901 (2006).

[25] H. Merdji, T. Auguste, W. Boutu, J.-P. Caumes, B. Carré, T. Pfeifer, A. Jullien, D. M. Neumark, and S. R. Leone, Opt. Lett. 32, 3134 (2007).

[26] L. E. Chipperfield, J. S. Robinson, J. W. G. Tisch, and J. P. Marangos, Phys. Rev. Lett. 102, 063003 (2009).

[27] C. Vozzi, F. Calegari, F. Frassetto, L. Poletto, G. Sansone, P. Villoresi, M. Nisoli, S. De Silvestri, and S. Stagira, Phys. Rev. A 79, 033842 (2009).

[28] A. Fleischer and N. Moiseyev, Phys. Rev. A 74, 053806 (2006). 
[29] M. B. Gaarde, P. Antoine, A. Persson, B. Carré, A. L'Huillier, and C.-G. Wahlstrom, J. Phys. B 29, L163 (1996).

[30] M. B. Gaarde, A. L'Huillier, and M. Lewenstein, Phys. Rev. A 54, 4236 (1996).

[31] E. Goulielmakis, V. Yakovlev, A. L. Cavalieri, M. Uiberacker, V. Pervak, A. Apolonski, R. Kienberger, U. Kleineberg, and F. Krausz, Science 317, 769 (2007).
[32] H.-C. Bandulet, D. Comtois, A. D. Shiner, C. Trallero-Herrero, N. Kajumba, T. Ozaki, P. B. Corkum, D. M. Villeneuve, J. C. Kieffer, and F. Légaré, J. Phys. B 41, 245602 (2008).

[33] P. Colosimo et al., Nat. Phys. 4, 386 (2008).

[34] A. Baltuska, T. Fuji, and T. Kobayashi, Phys. Rev. Lett. 88, 133901 (2002). 